DOI: $10.15393 /$ j3.art.2018.5470

The paper is presented at the conference "Complex analysis and its applications" (COMAN 2018), Gelendzhik - Krasnodar, Russia, June 2-9, 2018.

UDC 517.951

A. Biryuk, A. Svidlov, E. Silchenko

\title{
ON THE HEAT INTEGRAL IDENTITY FOR UNBOUNDED FUNCTIONS
}

\begin{abstract}
The well known heat integral identity in an unbounded strip is extended to a class of unbounded functions both at $x$ near infinity and $t$ near zero. Continuity of derivatives are relaxed to differentiability in the $L_{l o c}^{1}$-Sobolev sense.
\end{abstract}

Key words: heat operator, heat kernel

2010 Mathematical Subject Classification: $35 \mathrm{~K} 08$

1. Introduction. Let $\mathcal{L}$ denote the standard heat operator in Euclidean space $\mathbb{R} \times \mathbb{R}^{n}$, i. e., $\mathcal{L} u=u_{t}-\Delta u$. Under some restrictions there is the well-known integral representation identity that express the function $u$ through $\mathcal{L} u$ and the initial conditions $u_{0}=u(0, x)$. In particular, the classical result states that for $u(t, x) \in C\left([0, T) \times \mathbb{R}^{n}\right) \cap C^{1,2}\left((0, T) \times \mathbb{R}^{n}\right)$ if both $u$ and $\mathcal{L} u$ are bounded in the strip $(0, T) \times \mathbb{R}^{n}$, then there the integral identity (see $|2|$ )

$$
u(t, x)=\int_{\mathbb{R}^{n}} K(t, x-\xi) u_{0}(\xi) d \xi+\int_{0}^{t} \int_{\mathbb{R}^{n}} K(t-\tau, x-\xi) \mathcal{L} u(\tau, \xi) d \xi d \tau
$$

holds. Here $K$ stands for the fundamental solution to the heat operator (the heat kernel) and is given by

$$
K(t, x)= \begin{cases}\frac{1}{(2 \sqrt{\pi t})^{n}} \exp \left(-\frac{|x|^{2}}{4 t}\right), & t>0 \\ 0, & t \leqslant 0 .\end{cases}
$$

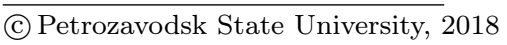

\section{(cc) EY-NC}


Among many applications, integral identity (1) is useful for analysis of solutions for various parabolic type equations, e.g., the Burgers equation $u_{t}-u_{x x}=-u u_{x}$, the Navier-Stokes equation

$$
\mathcal{L} u=-(u \cdot \nabla) u-\nabla p,
$$

and others. Unfortunately, the conditions above are too restrictive to cover many practical situations. For example, we are not allowed, formally, to consider the case of unbounded initial conditions. Moreover, for the Burgers, Navier-Stokes and similar equations, the case when the initial conditions are merely in $L^{\infty}$ can not be studied directly using (1), since the right-hand side contains the $x$-derivative of the function $u$, and, therefore, is unbounded near $t=0$.

In this paper we extend identity (1) to a much wider set of functions. In particular, the cases described above will be covered $|1|$.

2. Notations and the main result. Let $T$ be a positive number fixed throughout the paper. Let $L(\Omega)$ denote a set of measurable functions $f: \Omega \rightarrow \mathbb{R}$ and let

$$
M_{T}^{2}\left(\mathbb{R}^{n}\right)=\left\{f \in L\left(\mathbb{R}^{n}\right): \sup _{x \in \mathbb{R}^{n}}|f(x)| \exp \left(-\frac{|x|^{2}}{4 T}\right)<\infty\right\} .
$$

We denote the space of finite sums of the derivatives (in the sense of distributions) of these functions by $D_{T}^{\prime}$ :

$$
D_{T}^{\prime}\left(\mathbb{R}^{n}\right)=\left\{F \in D^{\prime}\left(\mathbb{R}^{n}\right): F=\sum_{k=1}^{m} \frac{\partial^{\left|\alpha_{k}\right|} f_{k}}{\partial x^{\alpha_{k}}}, f_{k} \in M_{T}^{2}\left(\mathbb{R}^{n}\right)\right\} .
$$

Here we have used the multi-index notation. Taking into account that the derivative of zeroth order is the function itself, we have a natural inclusion

$$
M_{T}^{2}\left(\mathbb{R}^{n}\right) \subset D_{T}^{\prime}\left(\mathbb{R}^{n}\right) .
$$

We note that for any $F \in D_{T}^{\prime}$, for any $\tau \in(0, T)$, and for any $\xi \in \mathbb{R}^{n}$ a function $g_{\tau, \xi}(x)=K(\tau, x-\xi)$ can be considered as a test function, since the value (the action) of $F$ on $g_{\tau, \xi}$ is well-defined as follows

$$
\left\langle F, g_{\tau, \xi}\right\rangle=\int_{\mathbb{R}^{n}} \sum_{k=1}^{m}(-1)^{\left|\alpha_{k}\right|} \frac{\partial^{\left|\alpha_{k}\right|} g_{\tau, \xi}}{\partial x^{\alpha_{k}}} f_{k} d x
$$


When it is does not lead to ambiguity, we allow the integral notation to denote the value of a distribution $F$ on a test function $g$ :

$$
\int_{\mathbb{R}^{n}} F(x) g(x) d x=\langle F, g\rangle .
$$

Now we are ready to list the assumptions for the main result.

Let $u:(0, T) \times \mathbb{R}^{n} \rightarrow \mathbb{R}$ be a continuous function, such that there exist partial derivatives $u_{t}, u_{x_{i}}, u_{x_{i} x_{j}} \in L_{l o c}^{1}\left((0, T) \times \mathbb{R}^{n}\right)$ in the Sobolev sense.

Let the functions $u$ and $\mathcal{L} u$ satisfy the following "moderate" growth conditions: for all $(t, x) \in(0, T) \times \mathbb{R}^{n}$, we have

$$
|u(t, x)| \leqslant c_{1}(t) \cdot \exp \left(\frac{|x|^{2}}{4 T}\right), \quad|\mathcal{L} u(t, x)| \leqslant c_{2}(t) \cdot \exp \left(\frac{|x|^{2}}{4 T}\right),
$$

where $c_{1}(t), c_{2}(t)$ satisfy the following condition for any $\varepsilon \in(0, T)$ :

$$
\int_{\varepsilon}^{T} c_{1}(t) d t<+\infty, \quad \int_{\varepsilon}^{T} c_{2}(t) d t<+\infty .
$$

Assume also, that there exists an initial condition $u_{0} \in D_{T}^{\prime}$, taken at $t=0$ in the following weak sense

$$
\lim _{t \rightarrow 0+} \int_{\mathbb{R}^{n}} K(\tau-t, \xi-x) u(t, x) d x=\left\langle u_{0}, g_{\tau, \xi}\right\rangle,
$$

for each $\tau \in(0, T), \xi \in \mathbb{R}^{n}$.

Theorem 1. Under the assumptions listed above the integral identity (1) holds true provided that the last integral in the right-hand side of (1) is understood as the improper Lebesgue integral near $\tau=0$ :

$u(t, x)=\int_{\mathbb{R}^{n}} K(t, x-\xi) u_{0}(\xi) d \xi+\lim _{\varepsilon \rightarrow+0} \int_{\varepsilon}^{t} \int_{\mathbb{R}^{n}} K(t-\tau, x-\xi) \mathcal{L} u(\tau, \xi) d \xi d \tau$. If $\int_{0}^{T} c_{2}(t) d t<\infty$, then the last integral in the right-hand side of (1) exists as the Lebesgue integral. 
3. Proof of Theorem 1. Define the following functions

$$
K_{\tau, \xi}(t, x)=K(t-\tau, x-\xi), \quad K_{\tau, \xi}^{*}(t, x)=K(\tau-t, \xi-x)
$$

and the following differential operators

$$
\mathcal{L} u=u_{t}-\Delta u, \quad \mathcal{L}^{*} u=-u_{t}-\Delta u .
$$

In $\left\{t \in \mathbb{R}, x \in \mathbb{R}^{n}\right\} \backslash\{(\tau, \xi)\}$, both functions $K_{\tau, \xi}$ and $K_{\tau, \xi}^{*}$ are $C^{\infty}$-smooth; so we have

$$
\mathcal{L}\left(K_{\tau, \xi}\right)=0 \quad \text { and } \quad \mathcal{L}^{*}\left(K_{\tau, \xi}^{*}\right)=0 .
$$

In what follows, we fix a point $(\tau, \xi) \in \mathbb{R} \times \mathbb{R}^{n}$, such that $\tau \in(0, T)$.

Let $\zeta_{N}(x)$ be a $C^{\infty}$ smooth function of the variable $x \in \mathbb{R}^{n}$. Since the function $u$ and its derivatives $u_{t}, u_{x_{i}}$, and $u_{x_{i} x_{i}}$ are assumed to be in $L_{l o c}^{1}$, the following identity holds in the $L_{l o c}^{1}\left((0, T) \times \mathbb{R}^{n} \backslash\{(\tau, \xi)\}\right)$ sense:

$$
\begin{aligned}
K_{\tau, \xi}^{*} \zeta_{N} \mathcal{L} u & =\left(K_{\tau, \xi}^{*} \zeta_{N} u\right)_{t}+K_{\tau, \xi}^{*} u \Delta \zeta_{N}+ \\
& +2 K_{\tau, \xi}^{*} \nabla \zeta_{N} \cdot \nabla u+\sum_{i=1}^{n}\left(\zeta_{N} u\left(K_{\tau, \xi}^{*}\right)_{x_{i}}-\left(\zeta_{N} u\right)_{x_{i}} K_{\tau, \xi}^{*}\right)_{x_{i}} .
\end{aligned}
$$

Indeed, for the smooth function $u$ this is a point-wise identity. The general case follows by approximation. We also note that if $\zeta_{N}(x)$ is compactly supported, then (2) makes sense in $L^{1}\left((\varepsilon, \tau-\varepsilon) \times \mathbb{R}^{n}\right)$ for any $\varepsilon \in(0, \tau / 2)$.

We choose smooth functions $\zeta_{N}(x)$ to satisfy $\zeta_{N}(x)=1$ for $|x| \leqslant N$, $\zeta_{N}(x)=0$ for $|x| \geqslant N+1$, and the condition that $\zeta_{N}, \nabla \zeta_{N}, \Delta \zeta_{N}$ are uniformly bounded by $N$ and $x$.

In a neighborhood of $[\varepsilon, \tau-\varepsilon] \times\{|x| \leqslant N+2\}$ the functions $\zeta_{N}$ and $K_{\tau, \xi}^{*}$ are infinitely smooth. Since all terms in (2) belong to $L^{1}([\varepsilon, \tau-\varepsilon] \times\{|x| \leqslant$ $\leqslant N+2\})$ and vanish for $|x| \geqslant N+1$, we can integrate (2) over $[\varepsilon, \tau-\varepsilon] \times \mathbb{R}^{n}$ and apply the Fubini theorem.

Omitting the standard mollification argument, we apply the divergence theorem for the last term in (2), as follows

$$
\begin{aligned}
& \int_{\varepsilon}^{\tau-\varepsilon} \int_{\mathbb{R}^{n}} \sum_{i=1}^{n}\left(\zeta_{N} u\left(K_{\tau, \xi}^{*}\right)_{x_{i}}-\left(\zeta_{N} u\right)_{x_{i}} K_{\tau, \xi}^{*}\right)_{x_{i}} d x d t= \\
& \quad=\int_{\varepsilon}^{\tau-\varepsilon} \int_{|x|<N+2} \sum_{i=1}^{n}\left(\zeta_{N} u\left(K_{\tau, \xi}^{*}\right)_{x_{i}}-\left(\zeta_{N} u\right)_{x_{i}} K_{\tau, \xi}^{*}\right)_{x_{i}} d x d t=
\end{aligned}
$$




$$
=\int_{\varepsilon}^{\tau-\varepsilon} \int_{\partial\{|x|<N+2\}} \sum_{i=1}^{n}\left(\zeta_{N} u\left(K_{\tau, \xi}^{*}\right)_{x_{i}}-\left(\zeta_{N} u\right)_{x_{i}} K_{\tau, \xi}^{*}\right) p_{i} d \sigma d t=0 .
$$

Here $\left(p_{1}, \ldots, p_{n}\right)$ is the outward pointing unit normal field of the sphere $\{|x|=N+2\}$. Thus, the integral of the last term in (2) vanishes.

Integrating by parts the third term in the right-hand side of (2), as follows:

$$
\int_{\mathbb{R}^{n}} K_{\tau, \xi}^{*} \nabla \zeta_{N} \cdot \nabla u d x=-\int_{\mathbb{R}^{n}} K_{\tau, \xi}^{*} \Delta \zeta_{N} u+\nabla K_{\tau, \xi}^{*} \cdot \nabla \zeta_{N} u d x
$$

and applying the fundamental theorem of calculus in $t$ variable for the first term in the right-hand side of (2), we finally arrive at

$$
\begin{gathered}
\int_{\varepsilon}^{\tau-\varepsilon} \int_{\mathbb{R}^{n}} K_{\tau, \xi}^{*} \zeta_{N} \mathcal{L} u d x d t=\int_{\mathbb{R}^{n}} K_{\tau, \xi}^{*}(\tau-\varepsilon, x) \zeta_{N}(x) u(\tau-\varepsilon, x) d x- \\
\int_{\mathbb{R}^{n}} K_{\tau, \xi}^{*}(\varepsilon, x) \zeta_{N}(x) u(\varepsilon, x) d x-\int_{\varepsilon}^{\tau-\varepsilon} \int_{\mathbb{R}^{n}}\left(K_{\tau, \xi}^{*} u \Delta \zeta_{N}+2 \nabla K_{\tau, \xi}^{*} \cdot \nabla \zeta_{N} u\right) d x d t .
\end{gathered}
$$

First we will take the limit of (3) as $N \rightarrow+\infty$, and then as $\varepsilon \rightarrow+0$. The following lemmas are needed to pass to the limit in (3) as $N \rightarrow+\infty$.

Lemma 1. For each $(\tau, \xi) \in(0, T) \times \mathbb{R}^{n}$ there is a number $\gamma_{1}(T, \tau, \xi)$ such that for any $t \in(0, \tau)$ we have

$$
\int_{\mathbb{R}^{n}} K_{\tau, \xi}^{*}(t, x) \exp \left(\frac{|x|^{2}}{4 T}\right) d x \leqslant \gamma_{1}(T, \tau, \xi) .
$$

Proof. An elementary calculation shows:

$$
\begin{aligned}
& \int_{\mathbb{R}^{n}} K_{\tau, \xi}^{*}(t, x) \exp \left(\frac{|x|^{2}}{4 T}\right) d x= \\
&=(4 \pi(\tau-t))^{-\frac{n}{2}} \int_{\mathbb{R}^{n}} \exp \left(\frac{|x|^{2}}{4 T}-\frac{|x-\xi|^{2}}{4(\tau-t)}\right) d x .
\end{aligned}
$$


Note that for any $a, b \in \mathbb{R}^{n}$ and for any $\lambda>0$ we have the inequality:

$$
|a+b|^{2} \leqslant(1+\lambda)|a|^{2}+\left(1+\frac{1}{\lambda}\right)|b|^{2} .
$$

Therefore, for any $\lambda>0$ we have

$$
|x|^{2}=|(x-\xi)+\xi|^{2} \leqslant(1+\lambda)|x-\xi|^{2}+\left(1+\frac{1}{\lambda}\right)|\xi|^{2} .
$$

Taking $\lambda=\frac{T-\tau}{2 \tau}$, we obtain

$$
\begin{array}{r}
\frac{|x|^{2}}{4 T}-\frac{|x-\xi|^{2}}{4(\tau-t)} \leqslant \frac{\frac{T+\tau}{2 \tau}(\tau-t)-T}{4 T(\tau-t)}|x-\xi|^{2}+\frac{T+\tau}{4 T(T-\tau)}|\xi|^{2} \leqslant \\
\leqslant-\frac{T-\tau}{8 T(\tau-t)}|x-\xi|^{2}+\frac{1}{2(T-\tau)}|\xi|^{2}
\end{array}
$$

We have used the inequalities $\tau-t<\tau$ and $T+\tau<2 T$ for the last step. We obtain

$$
\begin{aligned}
& \int_{\mathbb{R}^{n}} K_{\tau, \xi}^{*}(t, x) \exp \left(\frac{|x|^{2}}{4 T}\right) d x \leqslant \\
& \quad \leqslant(4 \pi(\tau-t))^{-\frac{n}{2}} \int_{\mathbb{R}^{n}} \exp \left(-\frac{T-\tau}{8 T(\tau-t)}|x-\xi|^{2}+\frac{1}{2(T-\tau)}|\xi|^{2}\right) d x .
\end{aligned}
$$

Now the identity

$$
\int_{\mathbb{R}^{n}} \exp \left(-c|x-\xi|^{2}\right) d x=\left(\frac{\pi}{c}\right)^{n / 2}
$$

completes the proof with $\gamma_{1}(T, \tau, \xi)=\left(\frac{2 T}{T-\tau}\right)^{n / 2} \exp \left(\frac{|\xi|^{2}}{2(T-\tau)}\right)$.

Lemma 2. For each $(\tau, \xi) \in(0, T) \times \mathbb{R}^{n}$ there is a number $\gamma_{2}(T, \tau, \xi)$ such that for any $t \in(0, \tau)$ we have

$$
\int_{\mathbb{R}^{n}}\left|\nabla K_{\tau, \xi}^{*}(t, x)\right| \exp \left(\frac{|x|^{2}}{4 T}\right) d x \leqslant \gamma_{2}(\tau, \xi) \cdot \frac{1}{\sqrt{\tau-t}} .
$$


Proof. Using the identity $\left|\nabla K_{\tau, \xi}^{*}(t, x)\right|=\frac{|x-\xi|}{2(\tau-t)} K_{\tau, \xi}^{*}(t, x)$ and arguing as in the proof of Lemma 1, we obtain

$$
\begin{aligned}
& \int_{\mathbb{R}^{n}}\left|\nabla K_{\tau, \xi}^{*}(t, x)\right| \exp \left(\frac{|x|^{2}}{4 T}\right) d x \leqslant \\
& \leqslant(4 \pi(\tau-t))^{-\frac{n}{2}} \int_{\mathbb{R}^{n}} \frac{|y|}{2(\tau-t)} \exp \left(-\frac{T-\tau}{8 T(\tau-t)}|y|^{2}+\frac{|\xi|^{2}}{2(T-\tau)}\right) d y,
\end{aligned}
$$

where $y=x-\xi$. Now the identity

$$
\int_{\mathbb{R}^{n}}|y| \exp \left(-c|y|^{2}\right) d y=\frac{\pi^{n / 2} \Gamma\left(\frac{n+1}{2}\right)}{c^{\frac{n+1}{2}} \Gamma\left(\frac{n}{2}\right)},
$$

where $\Gamma(s)=\int_{0}^{\infty} t^{s-1} e^{-t} d t$ is the Euler gamma function, completes the proof with $\gamma_{2}(T, \tau, \xi)=\left(\frac{2 T}{T-\tau}\right)^{\frac{n+1}{2}} \frac{\Gamma\left(\frac{n+1}{2}\right)}{\Gamma\left(\frac{n}{2}\right)} \exp \left(\frac{|\xi|^{2}}{2(T-\tau)}\right)$.

Since $|u(t, x)| \leqslant c_{1}(t) \exp \left(\frac{|x|^{2}}{4 T}\right)$, Lemma 1 implies

$$
\int_{\varepsilon}^{\tau-\varepsilon} \int_{\mathbb{R}^{n}}\left|K_{\tau, \xi}^{*} u \Delta \zeta_{N}\right| d x d t \leqslant \gamma_{1}(T, \tau, \xi) \sup \left|\Delta \zeta_{N}\right| \int_{\varepsilon}^{\tau-\varepsilon} c_{1}(t) d t<\infty .
$$

Using that $\frac{1}{\sqrt{\tau-t}} \leqslant \frac{1}{\sqrt{\varepsilon}}$ for $t \in[\varepsilon, \tau-\varepsilon]$, we conclude, by Lemma 2, that

$$
\int_{\varepsilon}^{\tau-\varepsilon} \int_{\mathbb{R}^{n}}\left|\nabla K_{\tau, \xi}^{*} \cdot \nabla \zeta_{N} u\right| d x d t \leqslant \gamma_{2}(T, \tau, \xi) \sup \left|\nabla \zeta_{N}\right| \int_{\varepsilon}^{\tau-\varepsilon} \frac{c_{1}(t)}{\sqrt{\tau-t}} d t<\infty .
$$

For any fixed $(t, x) \in[\varepsilon, \tau-\varepsilon] \times \mathbb{R}^{n}$ we have

$$
\lim _{N \rightarrow \infty} K_{\tau, \xi}^{*} u \Delta \zeta_{N}=0 \text { and } \lim _{N \rightarrow \infty} \nabla K_{\tau, \xi}^{*} \cdot \nabla \zeta_{N} u=0,
$$

because $\Delta \zeta_{N}(x)=0$ and $\nabla \zeta_{N}(x)=0$ for $N>|x|$. Therefore, we can apply the Lebesgue dominated convergence theorem and conclude that

$$
\lim _{N \rightarrow \infty} \int_{\varepsilon}^{\tau-\varepsilon} \int_{\mathbb{R}^{n}}\left(K_{\tau, \xi}^{*} u \Delta \zeta_{N}+2 \nabla K_{\tau, \xi}^{*} \cdot \nabla \zeta_{N} u\right) d x d t=0 .
$$


Hence, the limit of (3) as $N \rightarrow \infty$ gives us the following:

$$
\begin{aligned}
& \int_{\varepsilon}^{\tau-\varepsilon} \int_{\mathbb{R}^{n}} K_{\tau, x i}^{*} \mathcal{L} u d x d t= \\
& \quad=\int_{\mathbb{R}^{n}} K_{\tau, \xi}^{*}(\tau-\varepsilon, x) u(\tau-\varepsilon, x) d x-\int_{\mathbb{R}^{n}} K_{\tau, \xi}^{*}(\varepsilon, x) u(\varepsilon, x) d x .
\end{aligned}
$$

Replacing the domain of integration $[\varepsilon, \tau-\varepsilon] \times \mathbb{R}^{n}$ with $\left[\varepsilon_{1}, \tau-\varepsilon_{2}\right] \times \mathbb{R}^{n}$ and repeating the arguments, we obtain

$$
\begin{aligned}
& \int_{\varepsilon_{1}}^{\tau-\varepsilon_{2}} \int_{\mathbb{R}^{n}} K_{\tau, \xi}^{*} \mathcal{L} u d x d t= \\
& \quad=\int_{\mathbb{R}^{n}} K_{\tau, \xi}^{*}\left(\tau-\varepsilon_{2}, x\right) u\left(\tau-\varepsilon_{2}, x\right) d x-\int_{\mathbb{R}^{n}} K_{\tau, \xi}^{*}\left(\varepsilon_{1}, x\right) u\left(\varepsilon_{1}, x\right) d x .
\end{aligned}
$$

The continuity and boundedness assumptions on $u$ allow to conclude, that

$$
\lim _{\varepsilon_{2} \rightarrow 0} \int_{\mathbb{R}^{n}} K_{\tau, \xi}^{*}\left(\tau-\varepsilon_{2}, x\right) u\left(\tau-\varepsilon_{2}, x\right) d x=u(\tau, \xi) .
$$

Taking the limit of (4) and using Lemma 1, we conclude that

$$
u(\tau, \xi)=\int_{\mathbb{R}^{n}} K_{\tau, \xi}^{*}\left(\varepsilon_{1}, x\right) u\left(\varepsilon_{1}, x\right) d x+\int_{\varepsilon_{1}}^{\tau} \int_{\mathbb{R}^{n}} K_{\tau, \xi}^{*} \mathcal{L} u d x d t,
$$

where all integrals are understood in the Lebesgue sense. The assumptions of Theorem 1 allow to take limit as $\varepsilon_{1} \rightarrow 0$ and this completes the proof. In the case $\int_{0}^{T} c_{2}(t) d t<\infty$ Lemma 1 implies that the last term in the right-hand side of (1) exists as the Lebesgue integral.

Acknowledgment. This work was supported by the Dynasty Foundation and the Ministry of Education and Science of the Russian Federation (project 8.2321.2017/PCh).

The authors are grateful to B. E. Levitskii for research support at the Kuban State University. 


\section{References}

[1] Biryuk A., Svidlov A., Silchenko E. Use of Holder Norms for Derivatives Bounds for Solutions of Evolution Equations. International Conference "Geometrical analysis and its applications". (Volgograd, May, 30 June, 3), 2016. pp. 27-29 (in Russian).

[2] Mikhailov V.P. Partial Differential Equations. Second edition. Moscow, Nauka, 1983 (in Russian).

Received June 14, 2018.

Accepted September 21, 2018.

Published online September 26, 2018.

A. Biryuk

Kuban State University

149 Stavropolskaya str., Krasnodar 350040, Russia

E-mail: abiryuk@kubsu.ru

A. Svidlov

Kuban State University

149 Stavropolskaya str., Krasnodar 350040, Russia

E. Silchenko

Kuban State University

149 Stavropolskaya str., Krasnodar 350040, Russia 